\title{
Timing calibration of the LHAASO-KM2A electromagnetic particle detectors
}

\author{
Hongkui Lv ${ }^{* \dagger}$ \\ Institute of High Energy Physics, Chinese Academy of Sciences, Beijing 100049, China \\ E-mail: Ivhkeihep.ac.cn \\ Huihai He \\ Institute of High Energy Physics, Chinese Academy of Sciences, Beijing 100049, China \\ E-mail: hhh@ihep.ac.cn
}

\section{Xiangdong Sheng}

Institute of High Energy Physics, Chinese Academy of Sciences, Beijing 100049, China

E-mail: shengxdeihep.ac.cn

\section{Jia Liu}

Institute of High Energy Physics, Chinese Academy of Sciences, Beijing 100049, China

E-mail: jialiu@ihep.ac.cn

\begin{abstract}
The Large High Altitude Air Shower Observatory (LHAASO) is a multipurpose project focusing on high energy gamma-ray astronomy and cosmic ray physics. The $1 \mathrm{~km}^{2}$ array (KM2A) of this observatory will consist of 5242 electromagnetic particle detectors (EDs) and 1171 muon detectors (MDs). The remoteness and numerous EDs extremely demand a robust and automatic calibration procedure. In this paper, a self-calibration method which relies on the measurement of charged particles within the extensive air showers is proposed. The method is fully validated by Monte Carlo simulation and successfully applied in a KM2A prototype array experiment. The self-calibration method can be used to determine the detector time-offset constants at the subnanosecond level, which is adequate to meet the physical requirements of LHAASO experiment.
\end{abstract}

35th International Cosmic Ray Conference - ICRC2017

10-20 July, 2017

Bexco, Busan, Korea

\footnotetext{
*Speaker.

${ }^{\dagger}$ This work is supported by Natural Science Foundation of China (NSFC) under contacts No. 11375210.
} 


\section{Introduction}

The Large High Altitude Air Shower Observatory (LHAASO) will explore the gamma-ray sources with a sensitivity of $1 \% I_{\text {crab }}$ at energies above $50 \mathrm{TeV}$ [1]. The observatory will consist of an array with an area of $1 \mathrm{~km}^{2}$ (KM2A), which is the largest array in this observation, $78,000 \mathrm{~m}^{2}$ Water Cherenkov Detector Array (WCDA) and Wide Field Cherenkov telescope Array (WFCTA) (Fig. 1). The 5242 electromagnetic particle detectors (EDs) [2] in KM2A are designed to detect number densities and arrival times of EAS charged particles produced by the primary photons, from which the primary energy and direction of origin can be reconstructed. A reliable detector timing calibration is crucial to guarantee the optimal angular resolution, because direction reconstruction is directly related to the recorded particle arrival times. In particular, the precision in the calibration of the relative ED timings (i.e. detector time-offsets) should be better than $1 \mathrm{~ns}$.

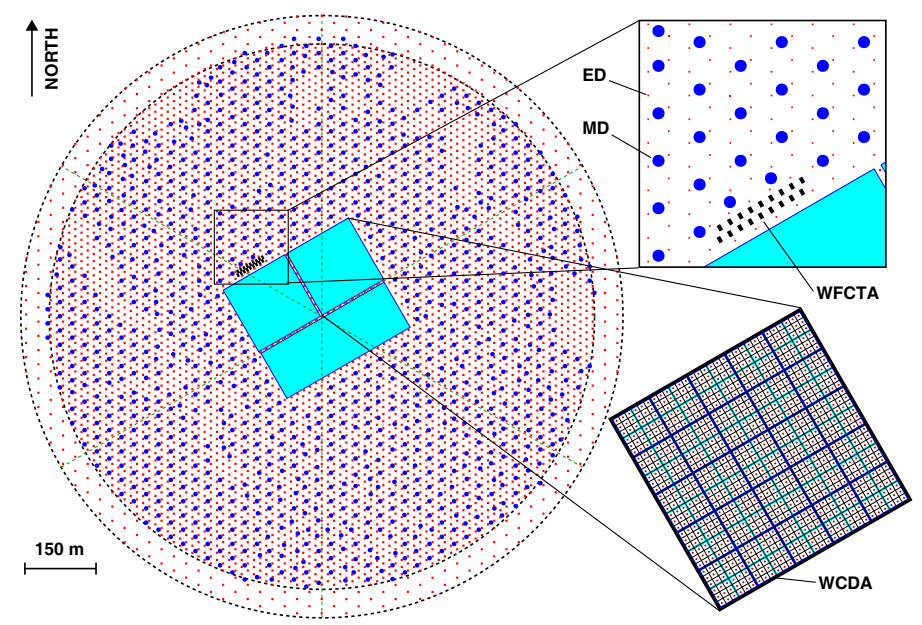

Figure 1: The layout of the LHAASO experiment.

For KM2A, which consists of thousands of EDs spread over $1 \mathrm{~km}^{2}$, global calibrating using the traditional method will require a significant amount of manpower and time. This will great affect the instrument stable operation and limit effective running time. Moreover, the EDs are deployed at high altitude and exposed to the natural environment, which has a big temperature variation of approximately $20^{\circ} \mathrm{C} /$ day. This variation may affect the detector performance and introduce a systematic bias in the physical results. Therefore, a fast, real-time self-calibrating and detector monitoring is required. The requirements for large-scale, high-precision and real-time calibration exceed the capability of traditional methods, making the global calibration a big challenge.

A automatic timing calibration technique named characteristic plane (CP) method is recently proposed to measure the relative time offset among the numerous of detector channels in the EAS experiment [3]. This method uses the EAS secondary charged particle as calibration beam and achieves real-time self-calibration without any additional devices. In a previous study on the ARGO-YBJ carpet array, energetic showers with more than 1500 hits (i.e. $10 \%$ of all units are fired) were selected as the calibration beam, corresponding a threshold energy of $\sim 10 \mathrm{TeV}$ and an event rate of $\sim 10 \mathrm{~Hz}$ [4]. The large size and high event rate guaranteed the large sample statistics and calibration speed. The experimental results showed that the time offsets on 1.5 day timescales 
could be corrected to a high precision of $0.45 \mathrm{~ns}$, which satisfies the KM2A requirement.

Whilst selection of large size showers is possible for a sparsely instrumented array in a very large area, in the case of KM2A, which covers an area about 100 times the size of the ARGO-YBJ carpet, the threshold energy can reach PeV level when $N_{\text {hit }}>1500$. Using such high energy shower suffers considerably from low event rate and low statistics, and these characteristics extremely limit the calibration speed and precision. Thus, we present an approach using the small showers $\left(N_{h i t}\right.$ $>20$, near KM2A trigger threshold) without any cut as calibration beam instead of large showers to overcome aforementioned limitations. The proposed approach enables a fast self-calibration of $\mathrm{KM} 2 \mathrm{~A}$, and its achievable precision is presented in this paper.

\section{LHAASO-KM2A electromagnetic particle detectors}

The KM2A is composed of 5242 EDs and 1171 MDs on a square grid with spacings of 15 $\mathrm{m}$ and $30 \mathrm{~m}$, respectively. The ED consists of four plastic scintillation tiles of $100 \mathrm{~cm} \times 25 \mathrm{~cm}$ $\times 2.5 \mathrm{~cm}$ each. The scintillation photons are detected by a 1.5 inch photomultiplier tube (PMT) through $2.7 \mathrm{~m}$ wavelength-shifting fibers embedded in the grooves in the scintillation tiles [5]. For each PMT, two output channels (anode and dynode outputs) with different gains and linearity performances are deployed to cover the large dynamic range [6]. The anode channel is used to detect signal charges up to 200 minimum ionization particles (MIPs), and the dynode channel is used to cover the range from $\sim 100$ MIPs to 10,000 MIPs.

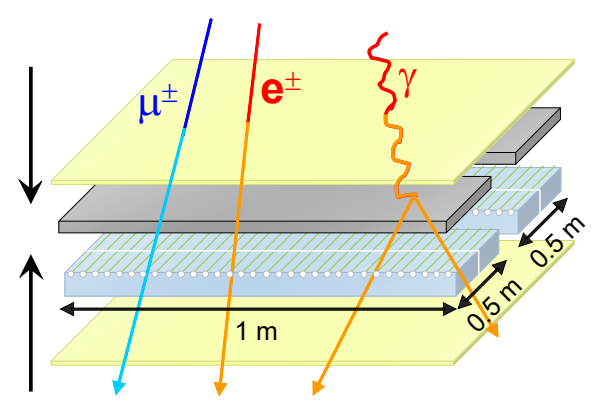

Figure 2: Schematic of a ED illustrating of 4 scintillation tiles coupled with wavelength-shifting fibers.

A very compact front-end electronics (FEE) is located just behind the PMT of each ED. Inside each FEE, an analog-to-digital converter (ADC) is used to integrate the signal charge, and a timeto-digital converter allows the measurement of hit times with sub-nanosecond precision [7]. Once an analogue PMT signal reaches an amplitude larger than a given threshold, the arrival time and charge of this signal are digitized by the FEE. Then, the digitized data is transmitted to the data acquisition system (DAQ) via optical fibers within a White Rabbit network. The White Rabbit is a precise timing and synchronous DAQ network, which can provide time synchronization within subnanosecond among all ED FEE nodes in the temperature variation of $50{ }^{\circ} \mathrm{C}$ [8]. The distributed digitization, data acquisition method and advanced timing system deployed within KM2A will enable enhanced angular resolution over current facilities. 


\section{Timing calibration}

An accurate reconstruction of the gamma-ray direction requires the precise determination of the arrival times of the EAS particles at various EDs. The main uncertainties on the measured hit times come from the time offset spread among the EDs. The time offset is measured as a cumulative effect of several contributing factors, such as the photon transmission time in the wavelengthshifting fibers and the transit time of PMTs. The typical value of the ED time offset is $\sim 40 \mathrm{~ns}$ and has a difference of a few nanoseconds between each individual detector. The systematic time differences lead to a decrease in angular resolution or more seriously introduce a bias in the reconstructed direction. Thus, the time offsets must be calibrated and corrected in the data periodically, to guarantee the synchronized global timing.

\subsection{Calibration principle}

The secondary particles of the shower front approximately sustain a conical shape, providing a common standard timing signal to calibrate the EDs. If the primary direction is known, the relative arrival time of secondary particles within the shower front can be exactly determined. In each EAS event, the expected hit time ( $\left.t^{\mathrm{real}}\right)$ of the i-th ED located at coordinates $\left(x_{i}, y_{i}\right)$ is given as follows:

$$
t_{i}^{\text {real }}=a^{\text {real }} \frac{x_{i}}{c}+b^{\text {real }} \frac{y_{i}}{c}+\alpha r_{i}+t_{0}
$$

where $a^{\text {real }}$ and $b^{\text {real }}$ are the components of the expected 'real' direction vector $(\mathrm{a}=\sin \theta \cos \phi$ and $\mathrm{b}$ $=\sin \theta \sin \phi(\theta$ and $\phi$ are the zenith and azimuth angles,respectively $)) ; r_{i}$ is the transverse distance of the $\mathrm{i}$-th ED from the shower core, $\alpha$ is the conicity coefficient, $c$ is the speed of light and $t_{0}$ is a fitting parameter of the direction reconstruction which corresponds to the arrival time of the EAS plane in the point of coordinates $(0,0)$.
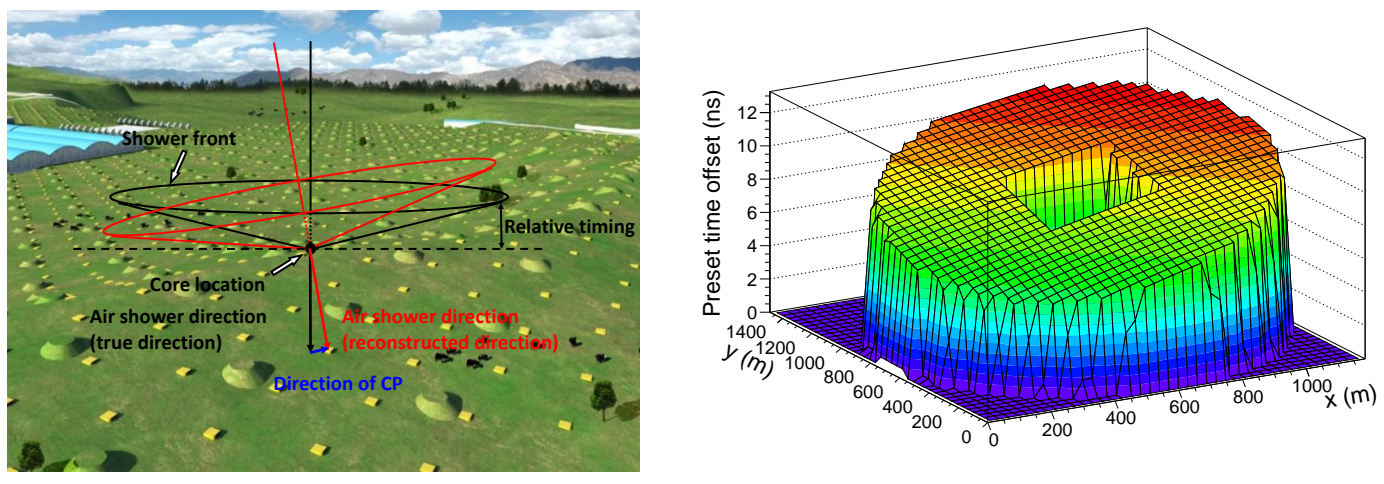

Figure 3: Schematic of a shower front and the CPFigure 4: Preset time offsets for all the EDs. These introduced by detector time offset. offsets are obtained from a curved surface equations $\left(\Delta t_{i}=\sqrt{\frac{x_{i}+y_{i}}{15}}\right)$.

According to the $\mathrm{CP}$ method, the detector time offsets introduce a systematic bias in the direction reconstruction, which can be described as a characteristic plane. This systematic error should 
be corrected before a timing calibration is performed. The correction can be performed by assuming an isotropic cosmic rays background. Assuming uniform azimuthal distribution, the mean value of the direction cosines (represented by $\left(\overline{a^{\text {real }}}, \overline{b^{\text {real }}}\right)$ ) for a set of EAS events is $(0,0)$, whilst the measured mean value (represented by $(\bar{a}, \bar{b})$ ) always slightly offsets from $(0,0)$ in practice. Thus, the systematic bias between the reconstructed direction and the true one is extracted from vector $(\bar{a}, \bar{b})$. Consequently, the time offset of the $\mathrm{i}$-th $\mathrm{ED}$ (represented by the time residual $\Delta t_{i}$ ) is determined as follows:

$$
\Delta t_{i}=t_{i}-t_{i}^{\text {real }}=t_{i}-\left[(a-\bar{a}) \frac{x_{i}}{c}+(b-\bar{b}) \frac{y_{i}}{c}+\alpha r_{i}+t_{0}\right]
$$

where $t_{i}$ and $t_{i}^{\text {real }}$ are the measured hit time of i-th ED and the expected 'real' one, respectively. $a$ and $b$ are the components of the reconstructed direction vector. The reconstructed direction is corrected using the direction correction parameters $(\bar{a}, \bar{b})$. In Equation 3.2, several parameters, namely (a,b), $\alpha$ and $t_{0}$, are derived from the direction reconstruction, and $r_{i}$ is obtained from the shower core reconstruction.

\subsection{Mente Carlo studies}

Prior to the actual experimental calibration, a complete timing calibration is performed using simulated showers, to verify the applicability of the CP method and estimate the calibration precision.

Approximately $2 \times 10^{6}$ EAS events are used for the timing calibration. The arrival direction of these EASs is isotropic, and the shower cores are spread randomly on an area larger than the array. For each EAS event, at least 20 triggered EDs are required to obtain a reliable reconstructed core and reduce the fluctuation of reconstructed direction, which is important in this calibration. These events are abundant in the KM2A data, and the corresponding event rate is approximately 1 KHz. The detector parameters used for simulating ED responses are identical to the experimental measuring values. The preset time offset of each ED is obtained from a curved surface equation, demonstrated in Fig. 4, and then artificially added into the detector response to distort the ED timing. Calculated results show that the global time offsets will introduce a systematic pointing error of $0.11^{\circ}$.

\subsubsection{Timing calibration with EASs}

The calibration method is an iterative procedure including the following steps:

1. A subset of EASs registered by the KM2A and within the criteria mentioned above, is selected.

2. The arrival direction of these EASs is reconstructed by $\chi^{2}$ minimization of the arrival times measured by the EDs with the expected ones from the conical front structure.

3. The direction correction parameters, namely, $(\bar{a}, \bar{b})$, are obtained from the mean value of the direction cosines of these EASs.

4. The hit time residuals of each ED are calculated according to Equation 3.2, event by event. 
5. A Gaussian function is fitted to the hit time residual distribution, the peak value of which is considered as the time correction to be applied to the hit times for a new iteration.

This procedure is repeated until the time corrections are small within the statistics fluctuation of the calibration method. In this study, the iteration times is artificially set to 6 .

\subsubsection{Calibration results}

As expected the mean direction cosines have the values of $\left(1.51 \times 10^{-3}, 1.31 \times 10^{-3}\right)$ in the first iteration, slightly offset from $(0,0)$. These two parameters are used to correct the reconstructed direction in the subsequent iterations. Consequently, the deviation vanishes fast and converges to the expected value of $(0,0)$ as the number of iterations increases. This result indicates that the systematic pointing error is effectively eliminated.

Good agreement is found between the preset true time offsets and obtained time offsets with this calibration procedure (Fig. 5). For illustration, the mean obtained time offset is set to the mean preassigned time offset. In practice, the absolute timing is not necessary in this calibration as long as the relative timing among EDs is aligned within the sub-nanosecond. A small bias is observed at EDs located at the edge of array, because the shower front is more likely a curved surface than a conical one. Nevertheless, this characteristic does not adversely affect the resulting overall calibration.

Comparing of the measured time offsets with the initial ones yields a direct prediction of the precision of the CP method. The distribution of time difference between the resulting time offsets and the preassigned time offsets is shown in Fig. 5. The root mean square of this distribution is $\sim 0.46 \mathrm{~ns}$, which is well within the required precision. To obtain this level of precision, at least $2 \times 10^{6}$ EAS events are needed to guarantee high direction correction accuracy better than $0.02^{\circ}$. Moreover, the number of hits of each ED should be larger than $10^{3}$ to ensure that the time residual is determined with a precision better than $0.1 \mathrm{~ns}$. Approximately $30 \mathrm{~min}$ of data collection is necessary to collect suitable calibration event statistics. Thus, very fast and high accurate calibration is performed using small-size shower.
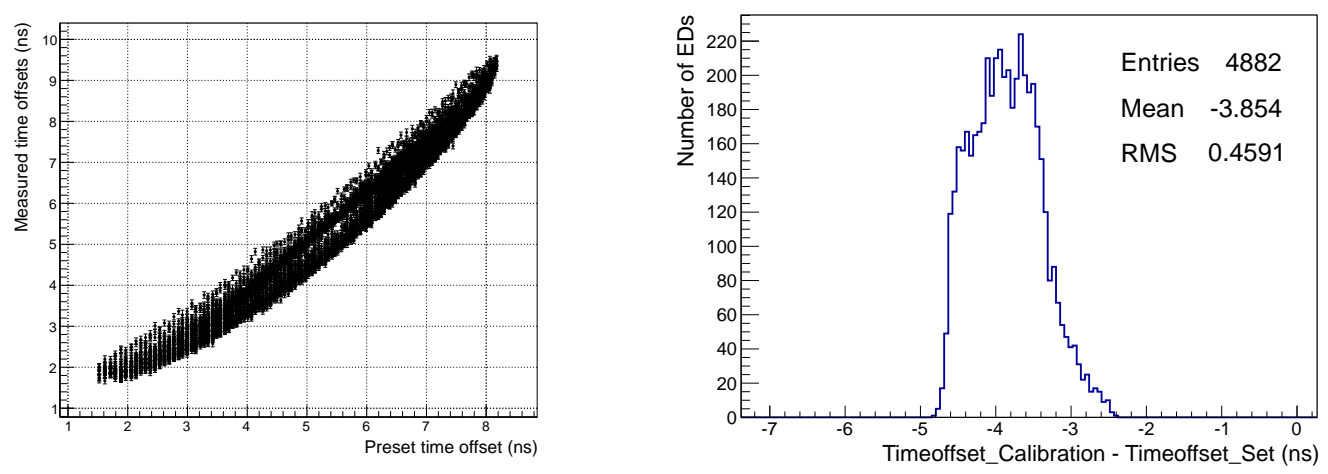

Figure 5: Left figure: Comparison of the 4883 EDs time offsets determined by applying the CP method on Monte Carlo simulations and the preassigned values introduced in order to distort the ED timing; Right figure: The observed time offsets differences for all EDs from their respective preset value. 


\subsection{Experimental calibration}

The calibration method is also validated by a KM2A prototype array, located at Yangbajing, Tibet at $4300 \mathrm{~m}$ a.s.l. The prototype array is composed of 39 EDs installed over an irregular grid with a spacing of $15 \mathrm{~m}$ and covers an area of approximately $110 \times 50 \mathrm{~m}^{2}$ (Fig. 6). The hardware used in this experiment is identical to those in the upcoming LHAASO-KM2A experiment. This array began to operate in October 2014 and has been running steadily for more than two years.

\subsubsection{Cross-check with the muon telescope system}

Muon telescope system can also be used to determine the time offsets between EDs. This system provides an independent calibration method to cross-check the results obtained from the $\mathrm{CP}$ method. Hence, a muon telescope made of three EDs with a vertical separation of $7 \mathrm{~cm}$ between them is established (Fig. 7). Each detector is sensitive to the passage of muons with a detection efficiency above $95 \%$. A 3-fold $300 \mathrm{~ns}$ coincidence between the three detectors selects almost all muons. Once background muons pass through the probe detector and the detector under calibration simultaneously, the difference in the response time between these two detectors can be determined. The relative time offsets are then measured by moving all EDs above the reference one. The hardware-level calibration method can achieve a high precision of $\sim 0.1$ ns under suitable statistics.

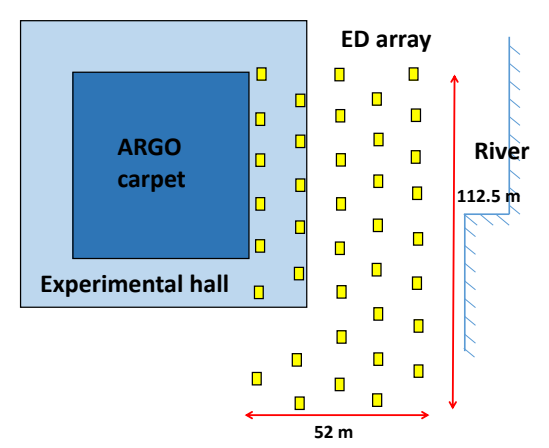

Figure 6: The layout of the KM2A prototype array. Figure 7: A muon telescope system composed of three EDs.

Using CP method, a software-level calibration is employed to determine the relative time offsets among the $39 \mathrm{EDs}$ in the prototype array. Excellent agreement is found between the time offsets obtained from CP method and those measured by the muon telescope (Fig. 8). The root mean square of the distribution of the differences between time offsets obtained from two independent measurements is $0.43 \mathrm{~ns}$ (Fig. 8), which is mainly contributed by the uncertainty of the software calibration. These results agree very well with the ones obtained from Monte Carlo simulation.

\subsubsection{Impact on the direction reconstruction}

The quality of direction reconstruction is observed after the corrected ED timing is applied. The chessboard method is used to study the angular resolution of the prototype array. The results shows that the angular resolution decreases from $2.64^{\circ}$ to $1.60^{\circ}$ after the ED timing is corrected. This result demonstrates a dramatic improvement of the array performance. 

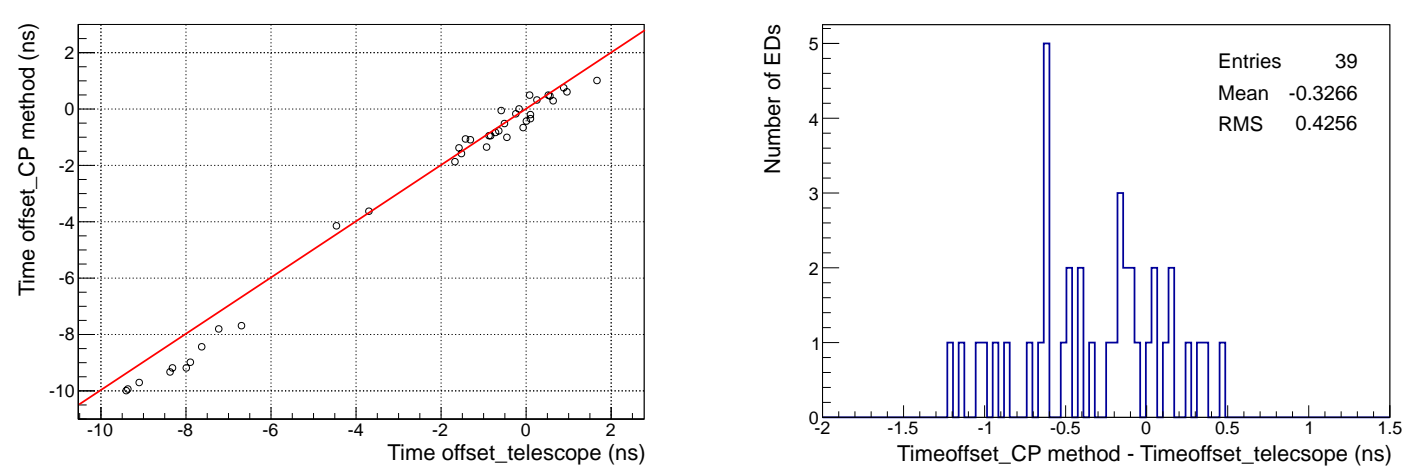

Figure 8: Left figure: Comparison of the 39 EDs time offsets measured using a muon telescope system and CP method; Right figure: Distribution of the differences between time offsets obtained from two independent measurements (muon telescope system and CP method).

\section{Conclusion}

The presence of hadronic component and the EAS they produced in every field observed can be very useful for calibration purposes. Calibration of ED timing using small size EAS events has been demonstrated as a robust and automatic approach that enables an independent calibration of many hardware technologies. Detector time offsets on hour timescales can be corrected to better than 0.5 $\mathrm{ns}$, which is within the KM2A performance requirements. In principle, the proposed method can be applied to other air shower physics experiments comprising huge number of detectors covering a large area.

\section{References}

[1] Cao Zhen, for the LHAASO Collaboration, Nucl. Instr. and Meth. A,742(2014),95-98.

[2] Liu Jia, et al., Chinese Physics C, 2014, 38(2):026001.

[3] H.H. He, et al., Astroparticle Physics, 27(2007),528-532.

[4] G. Aielli, et al., ARGO-YBJ Collaboration, Astroparticle Physics, 30(2009),287-292.

[5] Zhongquan Zhang, et al., Nucl. Instr. and Meth. A,845(2017),429-433.

[6] Hongkui Lv, et al., Nucl. Instr. and Meth. A,781(2015),34-38.

[7] Xiang Liu, et al., Chinese Physics C, 2016, 40(7):076101.

[8] Qiang Du, et al., Nucl. Instr. and Meth. A,732(2013),488-492. 